\title{
Vacuum-Field Catalysis: Accelerated Reactions by Vibrational Ultra Strong Coupling
}

\author{
Hidefumi Hiura $^{1 *} \&$ Atef Shalabney ${ }^{2}$ \\ ${ }^{1}$ System Platform Research Laboratories, NEC Corporation, 34 Miyukigaoka, Tsukuba \\ 305-8501, Japan \\ ${ }^{2}$ Physics and Optical Engineering Department, Braude College, Snunit St 51, Karmiel, \\ 2161002 Israel
}

In conventional catalysis, the reactants interact with specific sites of the catalyst in such a way that the reaction barrier is lowered by changing the reaction path, causing the reaction rate to be accelerated. Here we take a radically different approach to catalysis by ultra-strongly coupling the vibrations of the reactants to the infrared vacuum electromagnetic field. To demonstrate the possibility of such vacuum-field catalysis (or cavity catalysis), we have studied hydrolysis reactions under the vibrational ultra strong coupling (V-USC) of the OH stretching mode of water to a Fabry-Pérot microfluidic cavity mode. This results in a giant Rabi splitting energy ( $92 \mathrm{meV}$ ), indicating the system is in the V-USC regime. We have found that V-USC water enhances the hydrolysis reaction rate of cyanate ions by $10^{2}$-fold and that of ammonia borane by $10^{4}$-fold. This catalytic ability is found to depend upon the coupling ratio of the vibrational light-matter interaction. Given the vital importance of water for life and human activities, we expect that our finding not only offers an unconventional way of controlling chemical reactions by vacuumfield catalysis but also brings a fresh perspective to science and technology.

*Corresponding Author: Hidefumi Hiura

E-mail: $\quad$ h-hiura bq@, nec.com

Tel: $\quad$ + $\quad$ +818088401581 
Light-matter interactions have recently revealed an intriguing frontier for material science and chemistry ${ }^{1}$. In particular, it has been demonstrated that chemical reactions can be modified by coupling the reactant's vibrational transitions ${ }^{2-4}$. In such a case, a vibrational mode of a molecule is resonantly coupled to an optical mode of a cavity, thereby creating a pair of vibro-polaritons, i.e., light-matter hybrid (Fig. 1) ${ }^{1,5}$. Such vibrational strong coupling (V-SC) with an infrared (IR) electromagnetic vacuum field has been demonstrated both for solids and liquids in Fabry-Pérot (FP) cavities ${ }^{1-4,6-12}$. Moreover, theoretical model systems have been studied to understand how light-matter interactions affect the properties of the systems including chemical reactivity ${ }^{13-17}$.

In sharp contrast to earlier studies where the reaction rates decreased under V$\mathrm{SC}^{2,3}$, an accelerated reaction under V-SC has been reported recently: an $\sim 10$-fold rateincrease was observed for the solvolysis (ethyl acetate) of para-nitrophenyl acetate under $\mathrm{V}-\mathrm{SC}^{4}$. Here, for the first time, we demonstrate vacuum-field catalysis ${ }^{18}$ (or cavity catalysis $^{4}$ ) under vibrational ultra strong coupling (V-USC), showing an $\sim 10^{2}$-fold rateincrease for the hydrolysis $\left(\mathrm{H}_{2} \mathrm{O}\right)$ of cyanate ions $\left(\mathrm{OCN}^{-}\right)$and an $\sim 10^{4}$-fold rate-increase for the hydrolysis $\left(\mathrm{H}_{2} \mathrm{O}\right)$ of ammonia borane $\left(\mathrm{NH}_{3} \mathrm{BH}_{3}\right)$.

Figure 1 schematically outlines the vibrational light-matter coupling of water with an FP cavity. In brief, as shown in column (b), a pair of polaritonic states $\left(P_{-}\right.$and $\left.P_{+}\right)$ emerges when the $\mathrm{OH}$ stretch mode of water is vibrationally coupled with a given optical mode of an FP cavity under the resonant condition of $\omega_{0}=\omega_{c}$, where $\omega_{0}$ is the fundamental vibrational frequency of the $\mathrm{OH}$ stretch mode, and $\omega_{\mathrm{c}}$ is the frequency of the cavity mode. The difference in energy between $P_{+}$and $P_{-}$, so-called Rabi splitting energy, is given by the following equation ${ }^{1,5,6}$ :

$$
\hbar \Omega_{\mathrm{R}}=2 \sqrt{N} E d=2 \sqrt{N} \sqrt{\frac{\hbar \omega_{0}}{2 \varepsilon_{0} V}} \mathrm{~d} \sqrt{n_{\mathrm{ph}}+1}
$$

where $\hbar$ is the reduced Planck constant, $N$ is the number of coupled molecules, $E$ is the amplitude of the electric field of light, $d$ is the transition dipole moment of the molecules, $n_{\text {ph }}$ is the number of photons populating the cavity mode, $\varepsilon_{0}$ is the dielectric constant of vacuum, and $V$ is the mode volume. When $n_{\mathrm{ph}}=0, \hbar \Omega_{\mathrm{R}}$ has a finite value known as the 
vacuum Rabi splitting energy, which is due to the quantum fluctuation of IR vacuum field. The presence of such residual $\hbar \Omega_{\mathrm{R}}$ is crucial for vacuum-field catalysis because it ensures that vacuum-field catalysis works as a reaction booster even without any photons ${ }^{18}$. In addition, like conventional catalysis, vacuum-field catalysis requires no additional energy to function as a catalyst. The coupling ratio (or coupling strength) is defined as $\Omega_{R} / 2 \omega_{0}$ (refs. ${ }^{19,20}$ ). The boundary between the strong and ultra-strong coupling regimes is most often drawn at $\Omega_{\mathrm{R}} / 2 \omega_{0}=0.1$ (refs. ${ }^{19,20}$ ). Note that $\Omega_{\mathrm{R}} / 2 \omega_{0}$ is a decisive factor to define the catalytic power for vacuum-field catalysis under V-SC and V-USC as shown later.

To achieve V-SC and V-USC, the FP cavity is tuned to a molecular vibration by simply adjusting the distance between the two mirrors of the cavity, namely, the cavity length (see Supplementary Information: SI 1-3). Figure 2A compares the IR spectra of light-coupled $\mathrm{OH}(\mathrm{OD})$ oscillators in $\mathrm{H}_{2} \mathrm{O}\left(\mathrm{D}_{2} \mathrm{O}\right)$. The original $\mathrm{OH}(\mathrm{OD})$ mode is located at $\omega_{0}=3400(2500) \mathrm{cm}^{-1}$. These modes are coupled to the 9th and 7 th cavity modes, thereby forming pairs of vibro-polaritons $P_{9+} / P_{9-}$ and $P_{7+} / P_{7_{-}-}$, respectively. The measured $\Omega_{R}$ is approximately $740 \mathrm{~cm}^{-1}$ for pure $\mathrm{H}_{2} \mathrm{O}$ and $540 \mathrm{~cm}^{-1}$ for pure $\mathrm{D}_{2} \mathrm{O}$. The square root dependence is expected from equation (1), namely, $\Omega_{\mathrm{R}} / 2 \omega_{0}=A_{\mathrm{c}}\left(C / C_{0}\right)^{0.5}$, where $A_{\mathrm{c}}$ is a constant equal to $\Omega_{\mathrm{R}} / 2 \omega_{0}$ of the pure material. The least-squares fits (Fig. $2 \mathrm{~B}$ ) give $A_{\mathrm{c}}=$ 0.113 for $\mathrm{H}_{2} \mathrm{O}$ and 0.111 for $\mathrm{D}_{2} \mathrm{O}$, confirming that the regime belongs to ultra strong coupling $\left(\Omega_{\mathrm{R}} / 2 \omega_{0} \geq 0.1\right)$. Note that light-coupled water has the largest $\Omega_{\mathrm{R}}$ for vibrational light-matter coupling in molecular liquid ${ }^{8,21}$.

Next, the hydrolysis reaction under $\mathrm{V}$-USC of the $\mathrm{OH}(\mathrm{OD})$ stretch of $\mathrm{H}_{2} \mathrm{O}\left(\mathrm{D}_{2} \mathrm{O}\right)$ for the following reaction is studied:

$$
2 \mathrm{H}_{2} \mathrm{O}\left(2 \mathrm{D}_{2} \mathrm{O}\right)+\mathrm{OCN}^{-} \rightarrow \mathrm{CO}_{3}{ }^{2-}+\mathrm{NH}_{4}{ }^{+}\left(\mathrm{ND}_{4}{ }^{+}\right)
$$

where $\mathrm{H}_{2} \mathrm{O}\left(\mathrm{D}_{2} \mathrm{O}\right)$ acts both as a reactant and a solvent for the hydrolysis of cyanate ions $\left(\mathrm{OCN}^{-}\right)$. Figures $3 \mathrm{~A}$ and $3 \mathrm{~B}$ present the comparisons of temporal changes in IR spectra observed for the hydrolysis of $\mathrm{OCN}^{-}$in uncoupled $\mathrm{H}_{2} \mathrm{O}\left(\mathrm{D}_{2} \mathrm{O}\right)$ and under $\mathrm{V}-\mathrm{USC}$ at room temperature, respectively (see SI 1-4 for details). The insets of Figures 3A and 3B show that the absorption band of $\mathrm{OCN}^{-}$stretch under V-USC decreases much faster than they do with no V-USC. Since water was present in a large excess over $\mathrm{OCN}^{-}$in these solutions, 
the hydrolysis obeys a pseudo-first-order rate equation, $C=C_{0} \exp \left(-\kappa^{\prime} t\right)$, where $C$ is the time-dependent concentration of $\mathrm{OCN}^{-}, C_{0}$ is the initial concentration of $\mathrm{OCN}^{-}(2.00$ $\left.\mathrm{mol} \cdot \mathrm{dm}^{-3}\right), \kappa^{\prime}$ is the observed reaction rate constant of the hydrolysis (i.e., the product of the absolute rate constant $\kappa$ and the $\mathrm{H}_{2} \mathrm{O}\left(\mathrm{D}_{2} \mathrm{O}\right)$ concentration), and $t$ is the reaction time. Thus, the logarithmic plot (Fig. 3C) gives a rate constant under V-USC of $\mathrm{H}_{2} \mathrm{O}\left(\mathrm{D}_{2} \mathrm{O}\right), \kappa^{\prime}-$ $=6.4 \times 10^{-5}\left(1.6 \times 10^{-5}\right) \mathrm{s}^{-1}$, while that for the uncoupled $\mathrm{H}_{2} \mathrm{O}\left(\mathrm{D}_{2} \mathrm{O}\right)$ is $\kappa^{\prime} 0=5.6 \times 10^{-7}$ $\left(5.1 \times 10^{-7}\right) \mathrm{s}^{-1}$, demonstrating a 100 -fold enhancement in the absolute rate constant under V-USC for $\mathrm{H}_{2} \mathrm{O}$ and a 30-fold enhancement for $\mathrm{D}_{2} \mathrm{O}$. Figure $3 \mathrm{D}$ shows the dependence of $\kappa^{\prime}$ - on $\Omega_{\mathrm{R}}$ for light-coupled OH (OD) oscillators when tuned from $\Omega_{\mathrm{R}}=709$ (518) to $355(259) \mathrm{cm}^{-1}$ by diluting $\mathrm{H}_{2} \mathrm{O}\left(\mathrm{D}_{2} \mathrm{O}\right)$ with $\mathrm{D}_{2} \mathrm{O}\left(\mathrm{H}_{2} \mathrm{O}\right)$. $\kappa^{\prime}$ - increases exponentially with an increase of $\Omega_{\mathrm{R}}$ for both $\mathrm{OH}$ and OD light-coupled vibrations.

We next explore these accelerated reactions on the basis of a reaction kinetic model under IR vacuum field ${ }^{19}$. This kinetic model hypothesizes the reshape in reaction potential surface when going from the original potential to light-coupled potentials. In brief, when the Rabi splitting emerges, the force constant changes from $k_{0}$ to $k_{ \pm}$in accordance with the frequency change from $\omega_{0}$ to $\omega_{ \pm}$, where suffixes $0,+$, and - denote the original, $P_{+}$polariton, and $P_{-}$polariton, respectively. Because the force constant is also mechanically defined as the second derivative of the bottom of a vibrational potential, such changes in force constant reshape the corresponding reaction potential surfaces. Such reshapes in potential surface in turn alter reaction barrier heights. Among a pair of light-coupled potentials, namely, $P_{+}$and $P_{-}$potentials, almost all the reactants proceed via the $P$ - potential because it has a lower activation energy than the $P+$ potential.

The above kinetic model supposes that the reshape in reaction potential is driven by $\sqrt{N}$-multiplied collective quantities, IR vacuum field $(\sqrt{N} E)$ or transition dipole moment $(\sqrt{N} d)$, under vibrational light-matter coupling:, e.g., for the $\mathrm{OH}$ stretch of $\mathrm{H}_{2} \mathrm{O}$, equation (1) yields $\sqrt{N} E \approx 65 \mathrm{MV} \cdot \mathrm{cm}^{-1}$ or $\sqrt{N} d \approx 10^{5} \mathrm{D}$ (D: debye) when $n_{\text {ph }}$ is zero ${ }^{18}$. The former value is large enough to change reaction potentials, considering that electricfield catalysts (or electrostatic catalysts) can accelerate reactions up to $\sim 10^{5}$ rate-increase under a static electric field of $10 \sim 140 \mathrm{MV} \cdot \mathrm{cm}^{-1}$ (refs. ${ }^{22,23}$ ). 
Such qualitative picture of the vacuum-field catalysis can be analytically described by using the relative reaction rate constant $\left(\kappa_{-} / \kappa_{0}\right)$ as follows: by substituting next two formulae, $\omega_{ \pm} / \omega_{0}=\left(1 \pm \Omega_{\mathrm{R}} / 2 \omega_{0}\right)$ and $E_{ \pm} / E_{0}=\left(1 \pm \Omega_{\mathrm{R}} / 2 \omega_{0}\right)^{2}$, where $E_{ \pm}$is the activation energy via the $P_{ \pm}$potential under vibrational light-matter coupling and $E_{0}$ is the original activation energy, into the Eyring-Polanyi equation of the transition state theory ${ }^{24,25}$, we obtain the following equation ${ }^{18}$ :

$$
\frac{\kappa_{ \pm}}{\kappa_{0}}=\left(1 \pm \frac{1}{2} \frac{\Omega_{\mathrm{R}}}{\omega_{0}}\right) \exp \left[\left(-\frac{E_{0}}{k_{\mathrm{B}} T}\right)\left\{\left(1 \pm \frac{1}{2} \frac{\Omega_{\mathrm{R}}}{\omega_{0}}\right)^{2}-1\right\}\right]
$$

where $\kappa_{ \pm}$is the reaction rate constant via the $P_{ \pm}$potential under vibrational light-matter coupling, respectively, $\kappa_{0}$ is the original reaction rate constant, $k_{\mathrm{B}}$ is the Boltzmann constant, and $T$ is the absolute temperature. Note that in equation (2), $\kappa+$ and $\kappa$-correspond to the reaction rate constants for decelerated and accelerated reactions, respectively. Since all the reactions reported here are vacuum-field catalysed (rate-accelerated), we hereafter

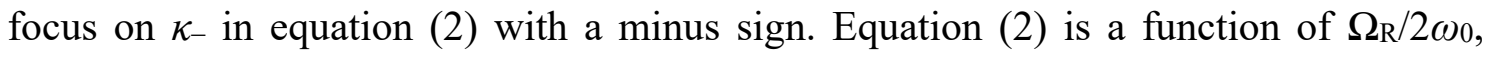
indicating that $\Omega_{\mathrm{R}} / 2 \omega_{0}$ is a key parameter for vacuum-field catalysis, as mentioned earlier. As $\Omega_{\mathrm{R}} / 2 \omega_{0}$ increases, $\kappa_{-} / \kappa_{0}$ increases exponentially. Note that the relation between $\kappa^{\prime}$ - and $\Omega_{\mathrm{R}}$ for light-coupled OH (OD) oscillators (Fig. 3D) can be well fitted by equation (2). Furthermore, the least-squares fitting gives $E_{0}=15.3 \mathrm{kcal} \cdot \mathrm{mol}^{-1}(0.66 \mathrm{eV})$, which agrees well with the literature value of $16 \mathrm{kcal} \cdot \mathrm{mol}^{-1}(0.69 \mathrm{eV})^{26}$ (see SI $1-4$ for details).

In the next example, a ten-thousand-fold acceleration in the hydrolytic dehydrogenation of ammonia borane $\left(\mathrm{NH}_{3} \mathrm{BH}_{3}\right)$ under $\mathrm{V}-\mathrm{USC}$ of $\mathrm{H}_{2} \mathrm{O}$ was observed and is shown in Figures 4A and 4B (see SI 1-5 for details). Equation (2) properly predicts that $\kappa_{-} / \kappa_{0}=\sim 10^{4}$ for this hydrolysis. These results imply that the above-mentioned kinetic model is at least applicable to hydrolysis reactions under V-SC to V-USC. Further experiments of vacuum-field catalysis have demonstrated that equation (2) can account rather well for several non-aqueous reactions under V-SC (see SI 2 and 3 with Figs. S1S6). From these results, we reckon that vacuum-field catalysis may be understood as a rate-accelerator empowered by giant IR vacuum field $(\sqrt{N} E)$ or giant transition dipole moment $(\sqrt{N} d)$ under vibrational light-matter coupling. 
Finally, we discuss about the decelerated reactions under V-SC reported so far $^{2,3}$. We suspect that the reactants in such a decelerated reaction may follow the $P+$ potential and their rate equation may be described by equation (2) with a plus sign, in which $\kappa_{+} / \kappa_{0}$ is always less than one ${ }^{18}$. In this scenario, the reaction path on the $P+$ potential must be solely opened. However, we have no particular idea of how the path on the $P$-potential is closed for such decelerated reactions. Thus, considering the myriad chemical reactions and their diverse nature, it will be necessary to compare more examples of different types of reactions in order to understand reactions under vibrational light-matter coupling.

To conclude, we anticipate that V-USC water can play a central role in vacuumfield catalysis because of the following reasons: (1) Water is of the essence in nature and in industry because it is utilized as reactants and media for numerous chemical reactions. (2) V-USC water possesses one of the largest $\Omega_{R}$ values among all the reactants. (3) Because water can be used as both a reactant and a solvent, V-USC water may maintain high reactivity through the endpoint of vacuum-field-catalysed reactions. Another advantage of water is that if water takes a form of microsphere in air, it may function as a self-resonator to generate a series of whispering gallery modes in an IR region. A simple estimate predicts that such airborne aqueous microdroplets can serve as a self-contained chemical reactor ideal for vacuum-field catalysis as long as the resonance condition of VUSC is fulfilled: their resonant diameters are distributed discretely but more densely than expected. To our surprise, not a few water-involved reactions have been in fact found to be dramatically promoted if confined in micrometer-sized water droplets ${ }^{27,28}$. The implications of such vacuum-field microreactors are as follows: what intrigues us the most is a possibility that nature might have already harnessed the principle of vacuumfield catalysis like an electric-field catalyst embedded in an enzymatic protein ${ }^{22}$. What if not all but a part of tiny dews of water — cloud, fog and marine aerosols — can be viewed as a naturally occurring vacuum-field reactor? Is there any chance that such a batch of microscopic "hydrospheres" have been and still are synthesising something beneficial and/or unfavourable to life and the global environment ${ }^{29}$ ? How much possibility was there that they could have played any active role in abiogenesis ${ }^{30}$ ?: how can a primordial sea mist be unrealistic, if enforced by the catalytic power of V-USC water? We envision that these pure but sound speculations will nurture a new dimension of science and technology. 


\section{References}

1. Ebbesen, T. W. Hybrid light-matter states in a molecular and material science perspective. Acc. Chem. Res. 49, 2403-2412 (2016).

2. Thomas, A. et al. Ground-state chemical reactivity under vibrational coupling to the vacuum electromagnetic field. Angew. Chem. Int. Ed. 55, 1-6 (2016).

3. Thomas, A. et al. Tilting a ground-state reactivity landscape by vibrational strong coupling. Science 363, 615-619 (2019).

4. Lather, J., Bhatt, P., Thomas, A., Ebbesen, T. W. \& George, J. Cavity catalysis by cooperative vibrational strong coupling of reactant and solvent molecules. Angew. Chem. Int. Ed. 58, 1-5 (2019).

5. Törmä, P. \& Barnes, W. L. Strong coupling between surface plasmon polaritons and emitters: a review. Rep. Prog. Phys. 78, 013901 (2014).

6. Shalabney, A. et al. Coherent coupling of molecular resonators with a microcavity mode. Nat. Comm. 6, 5981 (2015).

7. Shalabney, A. et al. Enhanced Raman scattering from vibro-polariton hybrid states. Angew. Chem. Int. Ed. 54, 7971-7975 (2015).

8. George, J. et al. Multiple Rabi splittings under ultrastrong vibrational coupling. Phys. Rev. Lett. 117, 153601 (2016).

9. Long, J. P. \& Simpkins, B. S. Coherent coupling between a molecular vibration and Fabry-Pérot optical cavity to give hybridized states in the strong coupling limit. ACS Photonics 2, 130-136 (2015).

10. Dunkelberger, A. D., Spann, B. T., Fears, K. P., Simpkins, B. S. \& Owrutsky, J. C. Modified relaxation dynamics and coherent energy exchange in coupled vibrationcavity polaritons. Nat. Comm. 7, 13504 (2016).

11. Xiang, B. et al. Two-dimensional infrared spectroscopy of vibrational polaritons. Proc. Natl. Acad. Sci. USA 115, 4845-4850 (2018).

12. Crum, V. F., Casey, S. R. \& Sparks, J. R. Photon-mediated hybridization of molecular vibrational states. Phys. Chem. Chem. Phys. 20, 850-857 (2018).

13. Flick, J., Ruggenthaler, M., Appel, H. \& Rubio, A. Atoms and molecules in cavities, from weak to strong coupling in quantum-electrodynamics (QED) chemistry. Proc. Natl. Acad. Sci. USA 114, 3026-3034 (2017). 
14. Galego, J., Garcia-Vidal, F. J. \& Feist, J. Many-molecule reaction triggered by a single photon in polaritonic chemistry. Phys. Rev. Lett. 119, 136001 (2017).

15. Martínez-Martínez, L. A., Ribeiro, R. F., Campos-González-Angulo, J. \& YuenZhou, J. Can ultrastrong coupling change ground-state chemical reactions? ACS Photonics 5, 167-176 (2018).

16. Galego, J., Climent, C., Garcia-Vidal, F. J. \& Feist, J. Cavity Casimir-Polder forces and their effects in ground-state chemical reactivity. Phys. Rev. X9, 021057 (2019).

17. Kéna-Cohen, S. \& Yuen-Zhou, J. Polariton chemistry: Action in the dark. ACS Cent. Sci. 5, 386-388 (2019).

18. Hiura, H. \& Shalabney, A. A reaction kinetic model for vacuum-field catalysis based on vibrational light-matter coupling. ChemRxiv. Preprint. https://doi.org/ 10.26434/chemrxiv.9275777.v1 (2019).

19. Ciuti, C. \& Bastard, G. Quantum vacuum properties of the intersubband cavity polariton field. Phys. Rev. B 72, 115303 (2005).

20. Bosman, S. J. et al. Multi-mode ultra-strong coupling in circuit quantum electrodynamics. npj Quantum Information 3, 46 (2017).

21. Hiura, H., Shalabney, A. \& George, J. Vibrational ultra strong coupling of water and ice. ChemRxiv. Preprint. https://doi.org/10.26434/chemrxiv.9808508.v1.

22. Fried, S. D., Bagchi, S. \& Boxer, S. G. Extreme electric fields power catalysis in the active site of ketosteroid isomerase. Science 346, 1510-1514 (2014).

23. Aragonès, A. C. et al. Electrostatic catalysis of a Diels-Alder reaction. Nature, 531, 88-91 (2016).

24. Eyring, H. The activated complex in chemical reactions. J. Chem. Phys. 3, 107 (1935).

25. Hänggi, P. \& Talkner, P. Reaction-rate theory: fifty years after Kramers. Rev. Mod. Phys. 62, 251-341 (1990).

26. Lister, M. W. Some observations on cyanic acid and cyanates. Can. J. Chem. 33, 426-440 (1955).

27. Fallah-Araghi, A et al. Enhanced chemical synthesis at soft interfaces: A universal reaction-adsorption mechanism in microcompartments. Phys. Rev. Lett. 112, 028301 (2014). 
28. Lee, J. K., Samanta, D., Nam, H. G. \& Zare, R. N. Micrometer-sized water droplets induce spontaneous reduction. J. Am. Chem. Soc. 141, 10585-10589 (2019).

29. Schwartz, S. Cloud Chemistry. Handbook of Weather, Climate, and Water. (eds Potter, T. D. \& Colman, B, R) (Wiley-Intersclence, 2003), Chap. 17, pp. 331-345.

30. Dobson, C. M., Ellison, G. B., Tuck, A. F. \& Vaida V. Atmospheric aerosols as prebiotic chemical reactors. Proc. Natl. Acad. Sci. USA 97, 11864-11868 (2000).

\section{Acknowledgements}

The authors acknowledge Professor Thomas W. Ebbesen for valuable discussions. H.H. thanks Jingwen Lu for assisting in the laboratory work.

\section{Author contributions}

A.S. instructed H.H. on how to perform V-SC experiments. H.H. planned the project of vacuum-field catalysis under V-USC. H.H acquired and analyzed the IR data. H.H. wrote the manuscript, and A.S. revised it. All authors contributed to discussions.

\section{Additional information}

Supplementary information is available for this paper.

\section{Data availability}

All data generated or analysed during this study are included in this published article (and its supplementary information files). 


\section{Figure 1}

(iii) Energy diagram

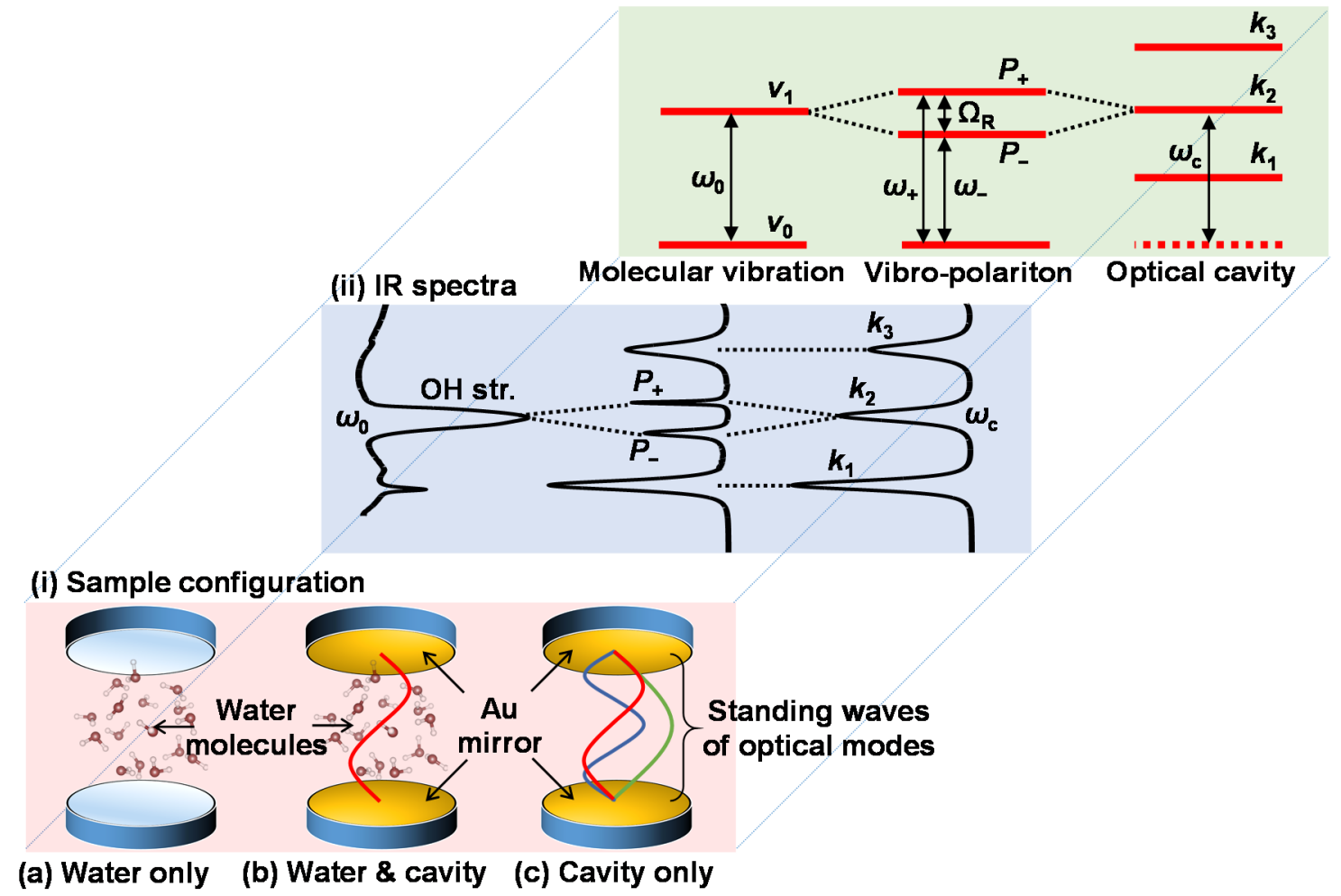

Fig. 1. Schematic overview of the vibrational light-matter coupling. The bottom, middle, and t rows show (i) sample configurations, (ii) IR transmission spectra, and (iii) energy diagrams, respectively. The left, middle, and right columns correspond to (a) water not in an FP cavity (just in an IR cell), (b) water in an FP cavity, and (c) an FP cavity without water, respectively. 
Figure 2
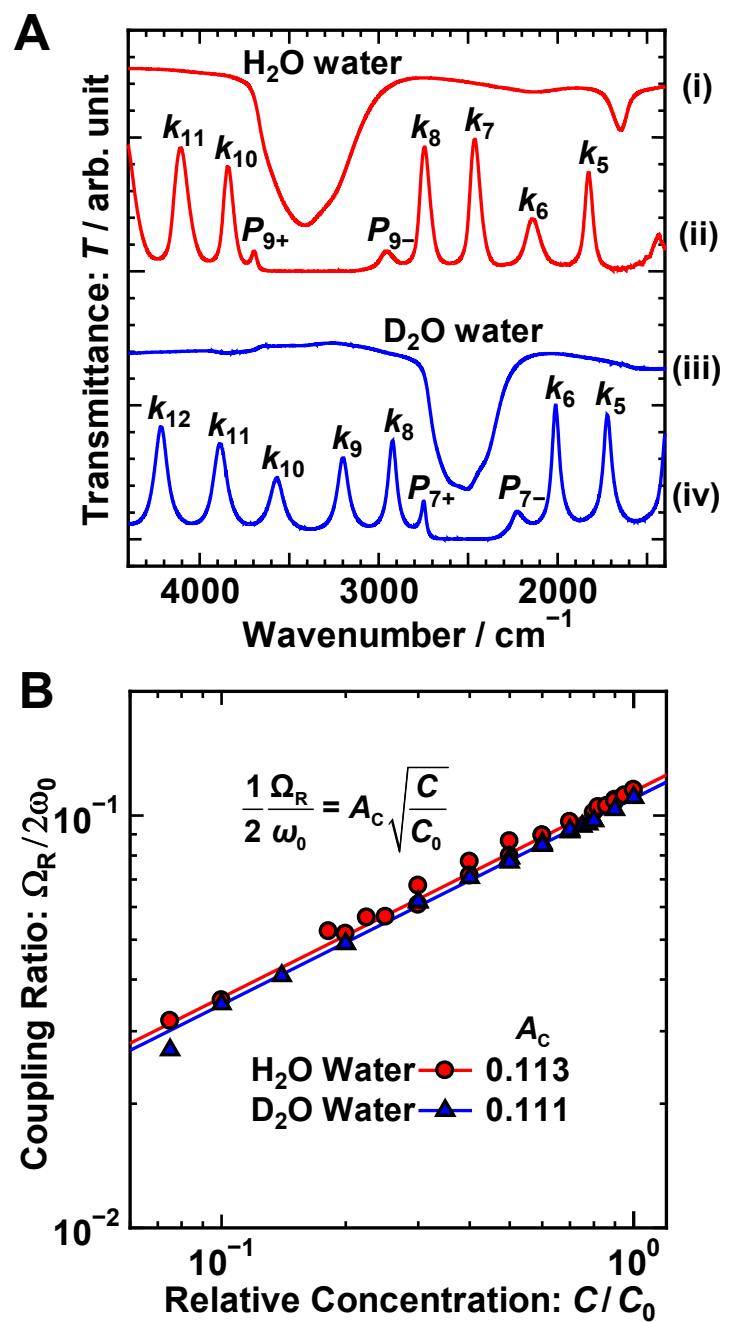

Fig. 2. V-USC of the $\mathrm{OH}(\mathrm{OD})$ stretch of $\mathrm{H}_{2} \mathrm{O}\left(\mathrm{D}_{2} \mathrm{O}\right)$. (A) Comparison of the IR spectra of uncoupled and light-coupled $\mathrm{H}_{2} \mathrm{O}\left(\mathrm{D}_{2} \mathrm{O}\right)$. (i) and (iii) are the IR spectra of pure $\mathrm{H}_{2} \mathrm{O}$ and $\mathrm{D}_{2} \mathrm{O}$, respectively, without V-USC, whereas (ii) and (iv) are those measured with V-USC, respectively. All the spectra are normalized to unity. (B) Relation between $\Omega_{R} / 2 \omega_{0}$ and relative concentration $\left(C / C_{0}\right)$, where $C_{0}$ denotes the concentration of pure $\mathrm{H}_{2} \mathrm{O}\left(\mathrm{D}_{2} \mathrm{O}\right)$, namely, $\sim 55.3(\sim 55.1) \mathrm{mol} \cdot \mathrm{dm}^{-3}$. 
Figure 3
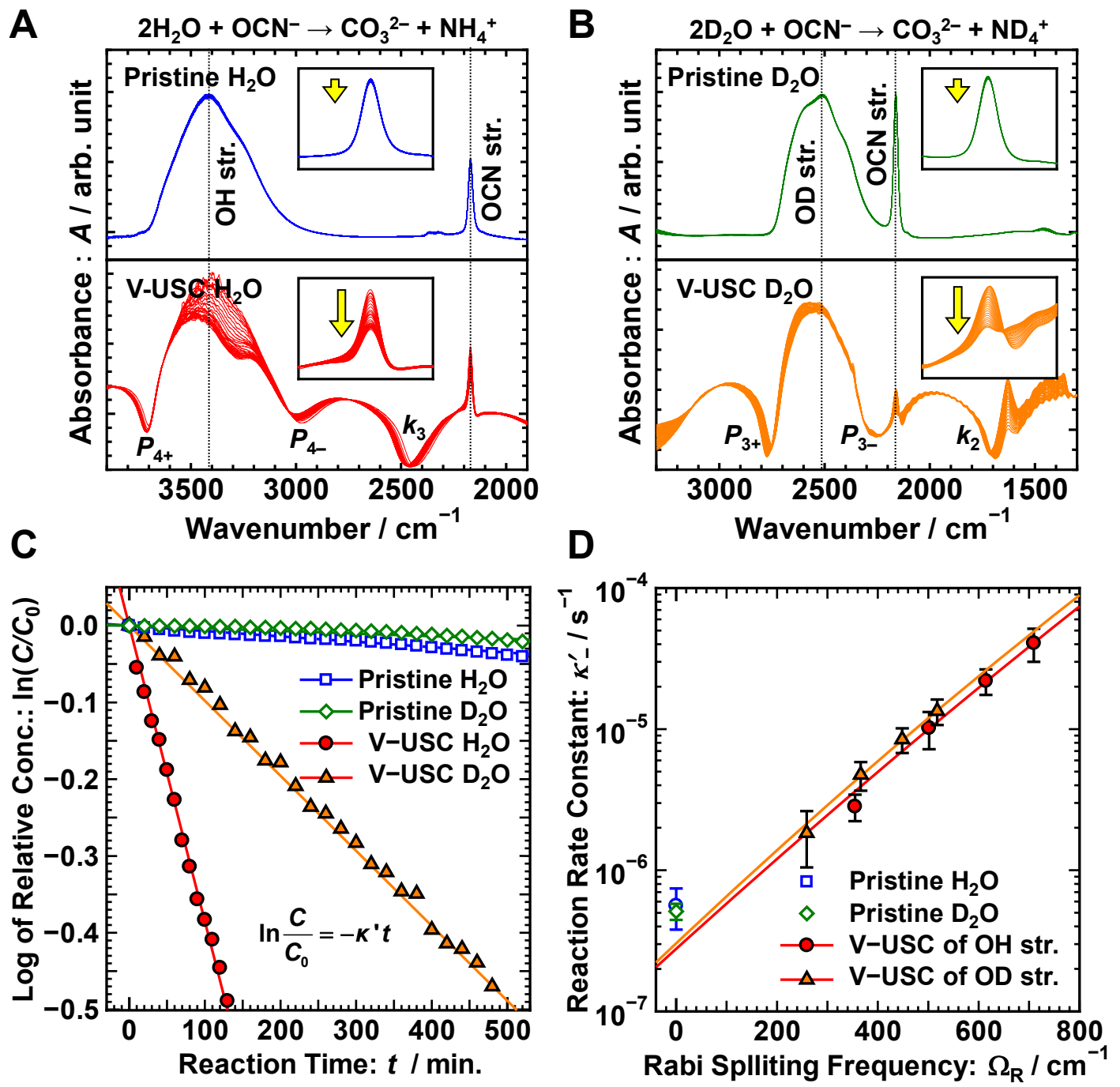

Fig. 3. Demonstration of the vacuum-field catalyst for $\mathrm{OCN}^{-}$under V-USC of water. $(A)$ and (B) Comparison of temporal changes in IR absorption spectra observed for the hydrolysis of $\mathrm{OCN}^{-}$with pristine and $\mathrm{V}-\mathrm{USC} \mathrm{H}_{2} \mathrm{O}$, and pristine and $\mathrm{V}$-USC $\mathrm{D}_{2} \mathrm{O}$. Insets show the magnified $\mathrm{O}=\mathrm{C}=\mathrm{N}$ stretch band during the reaction. (C) Comparison of the reaction profile for the hydrolysis of $\mathrm{OCN}^{-}$. (D) $\kappa^{\prime}-$ versus $\Omega_{R}$ on a semilogarithm scale for light-coupled $\mathrm{OH}$ and $\mathrm{OD}$ oscillators. Each bar denotes the standard errors of experimental values. The curves were drawn according to equation (2) by least-squares fitting. As a guide, $\kappa_{0}$ of pristine $\mathrm{H}_{2} \mathrm{O}\left(5.61 \pm 1.82 \times 10^{-7} \mathrm{~s}^{-1}\right)$ and $\mathrm{D}_{2} \mathrm{O}\left(5.12 \pm 0.68 \times 10^{-7} \mathrm{~s}^{-1}\right)$ were respectively plotted at $\Omega_{\mathrm{R}}=0 \mathrm{~cm}^{-1}$. 
Figure 4
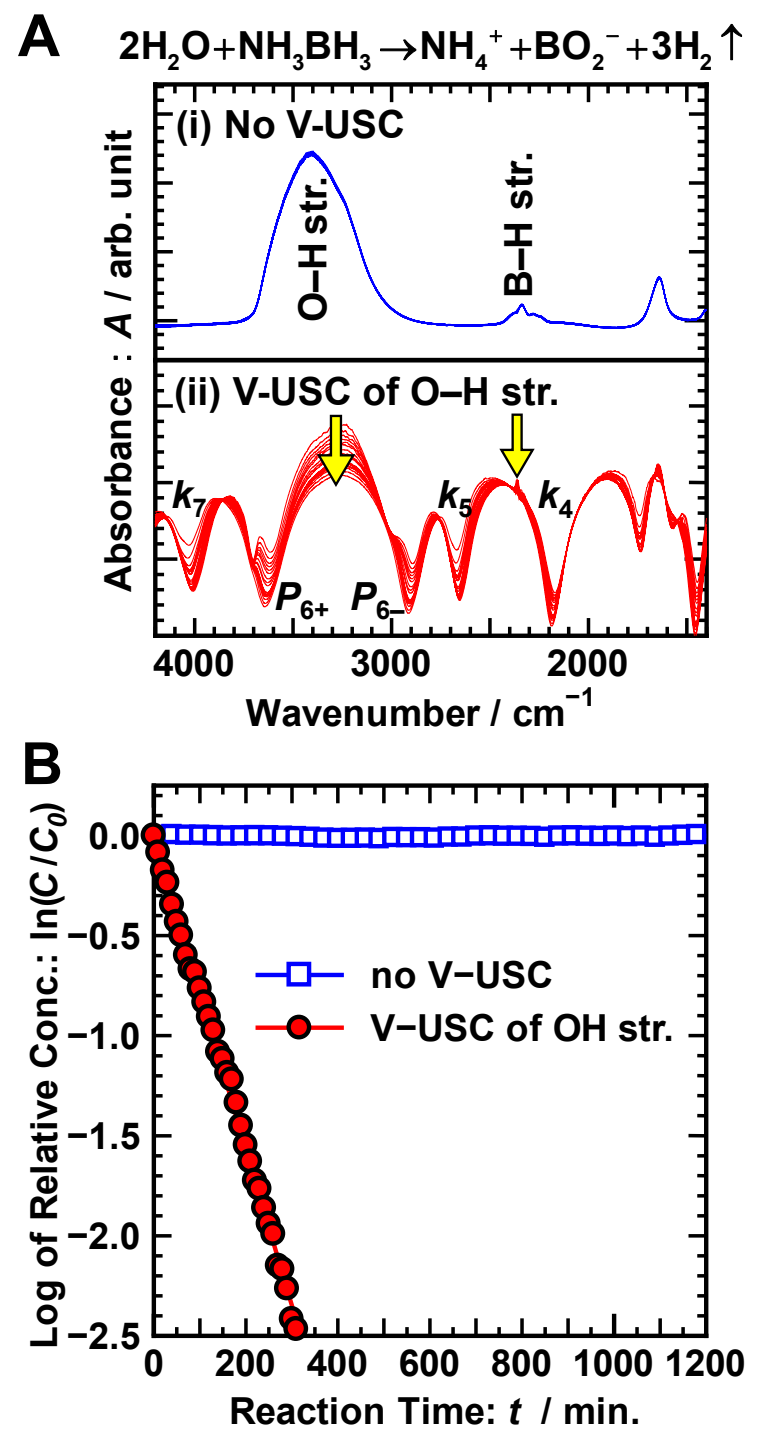

Fig. 4. Demonstration of the vacuum-field catalyst for $\mathrm{NH}_{3} \mathrm{BH}_{3}$ under V-USC of water. (A) Comparison of temporal changes of IR spectra observed for a hydrolysis of $\mathrm{NH}_{3} \mathrm{BH}_{3}$, (i) without V-USC, and (ii) with V-USC of the $\mathrm{OH}$ stretch. (B) Comparison of reaction profiles under the conditions of (i) and (ii). The pseudo-first-order rate constants were determined as follows: (i) $\kappa^{\prime} 0=1.29 \times 10^{-}$ ${ }^{8} \mathrm{~s}^{-1}$ (blue), and (ii) $\kappa^{\prime}-=1.29 \times 10^{-4} \mathrm{~s}^{-1}$ (red). 